\title{
QUEEN'S
UNIVERSITY
BELFAST
}

\section{Effects of filamentation instability on the divergence of relativistic electrons driven by ultraintense laser pulses}

Yang, X. H., Zhuo, H. B., Xu, H., Ge, Z. Y., Shao, F. Q., Borghesi, M., \& Ma, Y. Y. (2016). Effects of

filamentation instability on the divergence of relativistic electrons driven by ultraintense laser pulses. Physics of Plasmas, 23(10), [103110]. https://doi.org/10.1063/1.4966205

Published in:

Physics of Plasmas

Document Version:

Publisher's PDF, also known as Version of record

Queen's University Belfast - Research Portal:

Link to publication record in Queen's University Belfast Research Portal

Publisher rights

(C) 2016 AIP Publishing LLC

This article may be downloaded for personal use only. Any other use requires prior permission of the author and AIP Publishing. The following article appeared in Physics of Plasmas and may be found at (URL/link for published article abstract).http://scitation.aip.org/content/aip/journal/pop/23/10/10.1063/1.4966205

\section{General rights}

Copyright for the publications made accessible via the Queen's University Belfast Research Portal is retained by the author(s) and / or other copyright owners and it is a condition of accessing these publications that users recognise and abide by the legal requirements associated with these rights.

Take down policy

The Research Portal is Queen's institutional repository that provides access to Queen's research output. Every effort has been made to ensure that content in the Research Portal does not infringe any person's rights, or applicable UK laws. If you discover content in the Research Portal that you believe breaches copyright or violates any law, please contact openaccess@qub.ac.uk. 


\section{AIP $\left.\right|_{\text {Physics of }}$ \\ Plasmas}

\section{Effects of filamentation instability on the divergence of relativistic electrons driven by ultraintense laser pulses}

X. H. Yang, H. B. Zhuo, H. Xu, Z. Y. Ge, F. Q. Shao, M. Borghesi, and Y. Y. Ma

Citation: Physics of Plasmas 23, 103110 (2016); doi: 10.1063/1.4966205

View online: http://dx.doi.org/10.1063/1.4966205

View Table of Contents: http://scitation.aip.org/content/aip/journal/pop/23/10?ver=pdfcov

Published by the AIP Publishing

\section{Articles you may be interested in}

Enhanced electron injection in laser-driven bubble acceleration by ultra-intense laser irradiating foil-gas targets Phys. Plasmas 22, 083110 (2015); 10.1063/1.4927583

Density effects on collimation of energetic electron beams driven by two intense laser pulses

Phys. Plasmas 16, 043106 (2009); 10.1063/1.3112706

Evolution of the fastest-growing relativistic mixed mode instability driven by a tenuous plasma beam in one and two dimensions

Phys. Plasmas 13, 112110 (2006); 10.1063/1.2390687

Influence of betatron oscillation on filamentation instability in self-pinched hollow electron beam

Phys. Plasmas 10, 862 (2003); 10.1063/1.1544662

Magnetic instability by the relativistic laser pulses in overdense plasmas

Phys. Plasmas 7, 689 (2000); 10.1063/1.873853

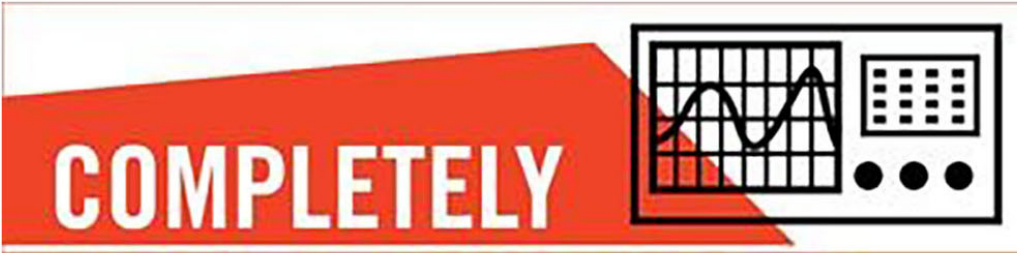

PHYSICS 


\title{
Effects of filamentation instability on the divergence of relativistic electrons driven by ultraintense laser pulses
}

\author{
X. H. Yang, ${ }^{1, a)}$ H. B. Zhuo, ${ }^{1}$ H. Xu, ${ }^{2}$ Z. Y. Ge, ${ }^{1}$ F. Q. Shao, ${ }^{1}$ M. Borghesi, ${ }^{3,4}$ and Y. Y. Ma ${ }^{1, b)}$ \\ ${ }^{1}$ College of Science, National University of Defense Technology, Changsha 410073, China \\ ${ }^{2}$ School of Computer Science, National University of Defense Technology, Changsha 410073, China \\ ${ }^{3}$ School of Mathematics and Physics, Queen's University of Belfast, Belfast BT7 1NN, United Kingdom \\ ${ }^{4}$ Institute of Physics of the ASCR, ELI-Beamlines Project, Na Slovance 2, 18221 Prague, Czech Republic
}

(Received 1 August 2016; accepted 13 October 2016; published online 27 October 2016)

Generation of relativistic electron (RE) beams during ultraintense laser pulse interaction with plasma targets is studied by collisional particle-in-cell simulations. A strong magnetic field with a transverse scale length of several local plasma skin depths, associated with RE current propagation in the target, is generated by filamentation instability in collisional plasmas, inducing a great enhancement of the divergence of REs compared to that of collisionless cases. Such an effect is increased with laser intensity and target charge state, suggesting that the RE divergence might be improved by using low-Z materials under appropriate laser intensities in future fast ignition experiments and in other applications of laser-driven electron beams. Published by AIP Publishing.

[http://dx.doi.org/10.1063/1.4966205]

\section{INTRODUCTION}

Ultraintense laser-driven REs have attracted great recent attention $^{1,2}$ due to their potential application in the areas of fast ignition laser fusion, ${ }^{3-6}$ ion acceleration by laser-plasma interaction, ${ }^{7,8}$ and production of ultrashort bright radiations. ${ }^{9}$ It is essential to characterize accurately the RE divergence for these applications.

Both the cause and characterization of the RE divergence are still not understood well, which are critically important to determine the RE energy deposition in targets. The divergence measured in the experiments usually increases with laser intensity ${ }^{10}$ though some discrepancy exists among the different diagnostic techniques because each one is dependent on different parameters. ${ }^{11}$ However, recent particle-in-cell (PIC) simulations show that the RE divergence is approximately linearly proportional to the preplasma scale length for a fixed laser intensity but is weakly dependent on the laser intensity for a fixed preplasma. ${ }^{12}$ For a normally incident laser, the electron injection angle $\theta_{i}=\tan ^{-1} \sqrt{2 /(\gamma-1)}$ is obtained from the electron's trajectory in an intense electromagnetic wave, ${ }^{13}$ where $\gamma$ is the electron relativistic factor. However, the scaling predicts a decrease of RE divergence as the laser intensity increases, which disaccords with both experimental and numerical results. Adam et al. ${ }^{14}$ found that the RE divergence is mainly induced by the large static magnetic fields generated in the laser-plasma interaction layer, arisen from the Weibel instability driven by a thermal anisotropy of the electrons. Furthermore, the full divergence of REs including a regular radial beam deviation and a random angular dispersion is reported recently, ${ }^{15}$ which are determined by the transverse component of the laser ponderomotive force and collisionless Weibel instability induced micro-magnetic fields, respectively. The Weibel/filamentation instability induced magnetic

\footnotetext{
${ }^{a)}$ Electronic mail: xhyang@nudt.edu.cn

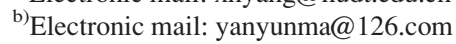

field will be saturated after a rapid linear growth due to magnetic trapping. ${ }^{16-18}$ In addition, magnetic field of $10^{4} \mathrm{~T}$ magnitude generated by the reflected laser in preplasma can also deflect the laser-driven REs. ${ }^{19}$

It is found that transverse temperature of beams can suppress the filamentation instability (FI) under certain conditions as it propagates in cold plasmas both in theoretical analysis and numerical simulations, but for a collisional plasma, the growth rate of instability will increase with plasma collision rate $^{20,21}$ or plasma resistivity (that is proportional to the collision rate), ${ }^{22}$ which can only be inhibited by the external magnetic field. ${ }^{20,23}$ In addition, collisional effects tend to attenuate the current FI for symmetric (where $n_{h}=n_{b}, n_{h}$, and $n_{b}$ are the relativistic and background electron density, respectively) or quasisymmetric counterstreaming while enhance it for extremely asymmetric counterstreaming (where $n_{h} \ll n_{b}$ ), ${ }^{24}$ which is in the context of laser-driven REs propagating in solid or compressed targets. Note that only the influence of magnetic field generated around the laser-plasma interaction region on the RE divergence and usually collisionless plasmas are considered in the previous studies. However, since the collision frequency of the background plasma can be comparable to the local plasma frequency in solid targets, the collisions of plasmas have to be considered properly as the RE propagates in it. ${ }^{25}$ Strong magnetic fields are ubiquitous during the RE beams propagating in the dense target due to growth of FI induced by plasma collisions. ${ }^{26-28}$ Such magnetic fields can significantly enhance or suppress the RE divergence, which is not yet fully understood and needs to be investigated in detail.

In this paper, angular distribution of laser-driven REs with laser intensity varying from $10^{18} \mathrm{~W} / \mathrm{cm}^{2}$ up to $5 \times 10^{20} \mathrm{~W} / \mathrm{cm}^{2}$ and target materials of copper $(\mathrm{Cu})$, aluminium ( $\mathrm{Al})$, and polymer $\left(\mathrm{CH}_{2}\right)$ are studied by collisional PIC simulations. It is found that a strong magnetic field is generated by the collisional FI during the REs propagating in collisional plasmas, inducing a great enhancement of the 
divergence of REs compared to that of collisionless cases. The divergence of REs increases with laser intensity and target charge state. This finding would be helpful for future fast ignition experiments and in other applications of laser-driven electron beams.

\section{SIMULATION MODEL}

To investigate the FI induced magnetic field on RE divergence in collisional plasmas, numerical simulations are performed using the relativistic collisional 2D3V PIC code $\mathrm{EPOCH},{ }^{29}$ which includes the binary collision model of charged particles proposed by Sentoku et al. ${ }^{30}$ The target consists of an initially neutral mixture of electrons and $\mathrm{Cu}$ ions with mass $m_{C u}=63.5 m_{p}$, where $m_{p}=1836 m_{e}$ is the proton mass. The charge state and maximum density of the ions are set to 15 and $28 n_{c}$, where $n_{c}=9.92 \times 10^{20} \mathrm{~cm}^{-3}$ is the critical density, corresponding to the laser wavelength $\lambda_{L}=1.06 \mu \mathrm{m}$. The plasma has an exponentially increasing density profile $n_{e}=5 n_{c} \exp ((x-10 \mu \mathrm{m}) / 1 \mu \mathrm{m})$ between $x=$ $6-10 \mu \mathrm{m}$ and then keeps the maximum electron density of $420 n_{c}$ between $x=10-25 \mu \mathrm{m}$. Note that $\sim 33 \%$ of the solid density of $\mathrm{Cu}$ ions is used in the simulations, in order to lower the computational costs of PIC simulations at the required resolution for full solid density. The initial temperature of the electrons and $\mathrm{Cu}^{15+}$ is $100 \mathrm{eV}$. The simulation box is $30 \mu \mathrm{m} \times 20 \mu \mathrm{m}$ with $3000 \times 2000$ cells. Each cell contains 50 numerical macroparticles per species in the simulations. A $p$-polarized laser pulse with a peak intensity of $I_{L}=10^{20} \mathrm{~W} / \mathrm{cm}^{2}$ is incident normally from the left boundary, corresponding to $a_{L}=e E_{L} / m_{e} c \omega_{L}=9.06$ for the dimensionless maximum amplitude of the laser electric field, where $e$ and $m_{e}$ are the electron charge and mass, $c$ is the light speed, and $E_{L}$ and $\omega_{L}$ are the electric field and frequency of the laser pulse, respectively. The spatial profiles of the laser are Gaussian, with spot radius $4 \mu \mathrm{m}$. The laser pulse rises up with a Gaussian profile over the first 15 fs to the peak intensity and stays at constant intensity for $105 \mathrm{fs}$. In order to suppress the numerical heating, the fifth-order interpolation scheme is employed to evaluate the currents. Periodic and absorbing boundary conditions are used for the transverse and longitudinal boundaries, respectively.

\section{RESULTS AND DISCUSSION}

Figure 1 shows the self-generated magnetic field and the electron kinetic energy density distributions for the case of a collisional $\mathrm{Cu}$ target. It can be seen that two types of large scale magnetic fields having distinct polarities are generated in the preplasma and around the solid target interface (i.e., $x=10 \mu \mathrm{m})$. The magnetic field generated in the preplasma is mainly arisen from $\nabla n_{e} \times \nabla T_{e}$ due to the gradients of electron density and temperature, whose magnitude can be estimated as ${ }^{31} B \sim 200\left(\frac{\tau}{\mathrm{ps}}\right)\left(\frac{k_{B} T_{e}}{\mathrm{keV}}\right)\left(\frac{L_{T}}{\mu \mathrm{m}}\right)^{-1}\left(\frac{L_{n}}{\mu \mathrm{m}}\right)^{-1} \mathrm{~T}$, where $T_{e}$ is the electron temperature, $\tau$ is the pulse duration, and $L_{T}$ and $L_{n}$ are the (transverse) temperature and (longitudinal) density gradient, respectively. $L_{T}$ and $L_{n}$ can be estimated from the electron temperature and density distributions, which are around $5.5 \mu \mathrm{m}$ and $1.4 \mu \mathrm{m}$, respectively, and the electron temperature is $3.03 \mathrm{MeV}$ (see Fig. 5(a)) in the preplasma. Thus, the magnitude of $B$ is $9.4 \times 10^{3} \mathrm{~T}$, which is very close to the simulation results. The field around the solid target interface is driven by temporal variations in the ponderomotive force and can be estimated by $\nabla^{2} B \sim \nabla n_{e} \times \nabla I,{ }^{32}$ where $I$ is the laser intensity. In addition to the large scale magnetic fields, microscale magnetic field with periodical distribution is also observed in the dense target, whose magnitude can reach $1.5 \times 10^{4} \mathrm{~T}$. The fields is associated with the RE propagation in the target, as shown in Figs. 1(c) and 1(d), in which the REs are beamed into the target and heat the target rapidly.
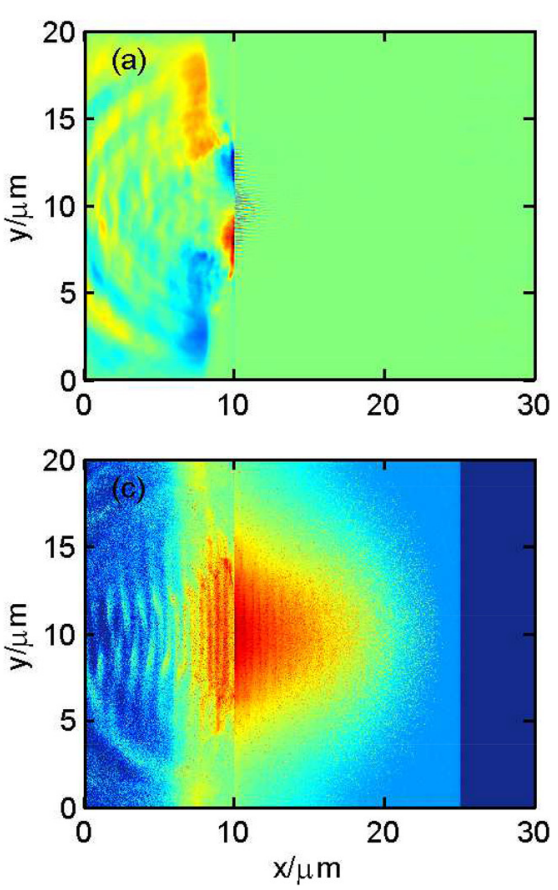
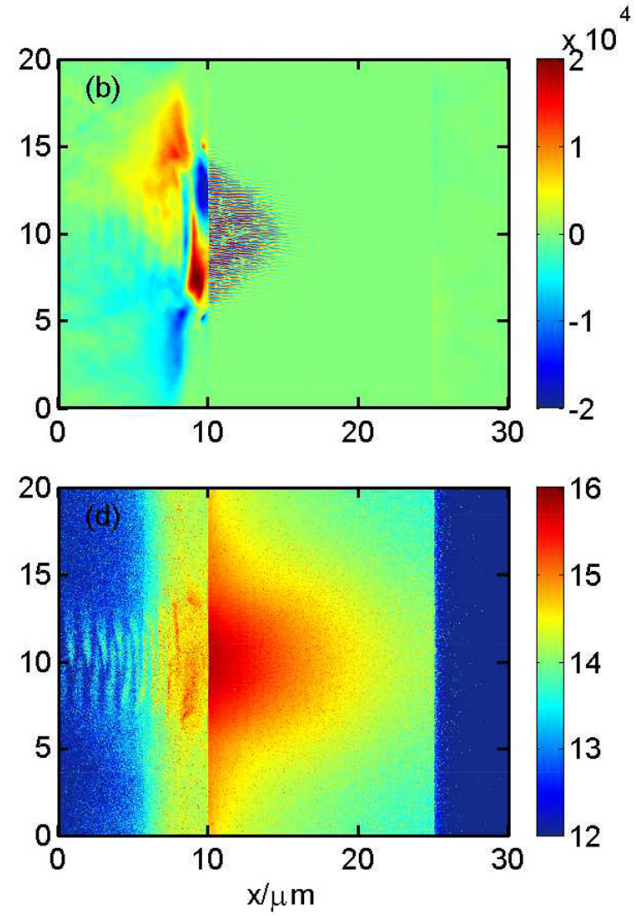

FIG. 1. Distributions of the selfgenerated magnetic field $\left(B_{z}\right)[(a)$ and (b)] and $\log _{10}$ of kinetic energy density of the electrons [(c) and (d)] at $\mathrm{t}=100$ fs $[(a)$ and (c)] and $160 \mathrm{fs}[(\mathrm{b})$ and (d)] for the case of a collisional $\mathrm{Cu}$ target, respectively. The magnetic field is in units of $\mathrm{T}$ and the kinetic energy density is in units of $J / \mathrm{m}^{3}$ (the same as in the other figures). 

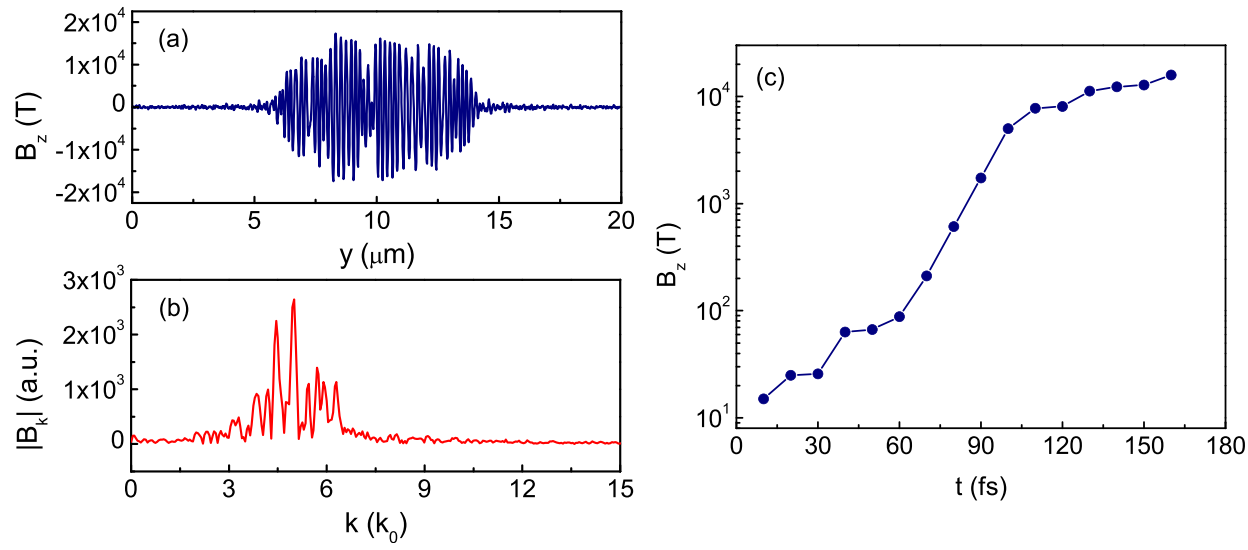

FIG. 2. Profile of $B_{z}$ along the $y$ direction at $x=11 \mu \mathrm{m}$ at $\mathrm{t}=160 \mathrm{fs}$ (a), which is averaged over $1 \mu \mathrm{m}$ along the $x$ direction. The corresponding frequency spectrum $\left|B_{k}\right|$ of $B_{z}$ (b). Evolution of $B_{z}$ with time in the front surface of the solid target around $(x=11 \mu \mathrm{m}, y=8 \mu \mathrm{m})(\mathrm{c})$
The kinetic energy density of the electrons can be greater than $10^{16} \mathrm{~J} / \mathrm{m}^{3}$.

The profile of $B_{z}$ along the $y$ direction at $x=11 \mu \mathrm{m}$ and the corresponding frequency spectrum $\left|B_{k}\right|$ of $B_{z}$ are presented in Figs. 2(a) and 2(b). It is shown that the peak of the wave number of the magnetic field is $\sim 5 k_{0}$, where $k_{0}=2 \pi / 1 \mu \mathrm{m} \approx k_{L}, k_{L}$ is the wave number of the laser pulse. The local plasma skin depth $\lambda_{p}=2 \pi c / \omega_{p}=0.049 \lambda_{L}$, where $\omega_{p}=\left(4 \pi n_{e} e^{2} / m_{e}\right)^{1 / 2}$ and $n_{e}$ are the local electron frequency and density. Thus, the transverse scale length of the magnetic field is $\sim 4 \lambda_{p}$. The growth rates of modes whose wavelength is comparable to the plasma skin depth are suppressed and the spectral peak of the growth rate shifts to long wavelength modes compared to that of collisionless case (not shown for brevity), similar to the previous theoretical and numerical results. $^{24,33}$ Thus, magnetic fields with relatively large structures are observed in the collisional plasmas, as shown in Figs. 1(b) and 2(a).

Figure 2(c) shows the evolution of self-generated magnetic field $B_{z}$ with time in the front surface of the solid target. It is seen that the magnetic field grows slowly before $t=60 \mathrm{fs}$; however, it grows rapidly with a linear growth rate as the REs propagates into the target during $t=60-100 \mathrm{fs}$. The maximum growth rate of the magnetic field is estimated to be $\Gamma_{\max }=1.07 \times 10^{14} / \mathrm{s} \approx 0.03 \omega_{p}$. Assuming that the only role of collisions is to slow down the REs, for simplicity, the maximum growth rate of FI for a "cold" beam propagating in collisionless plasmas is employed ${ }^{34}$

$$
\Gamma \sim \beta_{h} \sqrt{\frac{\kappa}{\gamma_{h}}},
$$

where $\beta_{h}=v_{h} / c, v_{h}$ is the RE velocity along the laser propagation axis, $c$ is the light speed in vacuum, $\gamma_{h}$ is the Lorentz factor of REs, and $\kappa=n_{h} / n_{b}$ is the beam to plasma density ratio. From the RE temperature here (3.03MeV, see Fig. 5(a)), we get $\gamma_{h} \approx 6.93$ and $\beta_{h}=0.9895$. The laser absorption efficiency here is $\alpha=0.42$, and the RE density can be obtained by conservation of energy flux $\alpha I_{L}=n_{h} v_{h} T_{h}$, which leads to a RE density of $n_{h}=2.93 \times 10^{21} \mathrm{~cm}^{-3}$. Thus, $\kappa=0.004$ and $\Gamma=0.024 \omega_{p}$, which are comparable to the simulation results. It is seen that the field becomes saturated nonlinearly with a magnitude of $\sim 1.5 \times 10^{4} \mathrm{~T}$ after $t=120 \mathrm{fs}$. Such nonlinear saturation appears as the gyroradius of REs becomes of the order of the modulation wavelength, i.e., $r_{L} \sim \lambda_{F}$, namely, magnetic trapping, ${ }^{16,17}$ leading to a saturation magnetic field amplitude

$$
B_{s}=\frac{\gamma_{h} m_{e} v_{y}}{e \lambda_{F}}
$$

where $v_{y} \approx 0.4 c$ is the transverse velocity of REs that is estimated from the RE temperature and divergence (i.e., $43.4^{\circ}$, see Fig. 5(b)), $\lambda_{F}=4 \lambda_{p}$ is applied here. Thus, the saturated magnetic field can be estimated to be $B_{s}=2.25 \times 10^{4} \mathrm{~T}$, which has the same order with that observed in the PIC simulation.

In order to understand better the filamentation instability observed here, we show the fast Fourier transform of $B_{z}$ at $\mathrm{t}=100 \mathrm{fs}$ and $160 \mathrm{fs}$ in Fig. 3. It can be seen that transverse modes appear with time evolution, and a clear maximum wave number of around $k_{y}=5 k_{0}$ is observed, which persists that in Fig. 2. In addition, the peak spread widely in $k_{x}-k_{y}$ space, indicating that the modes are not purely transverse but oblique. This is consistent with the theoretical results ${ }^{35,36}$
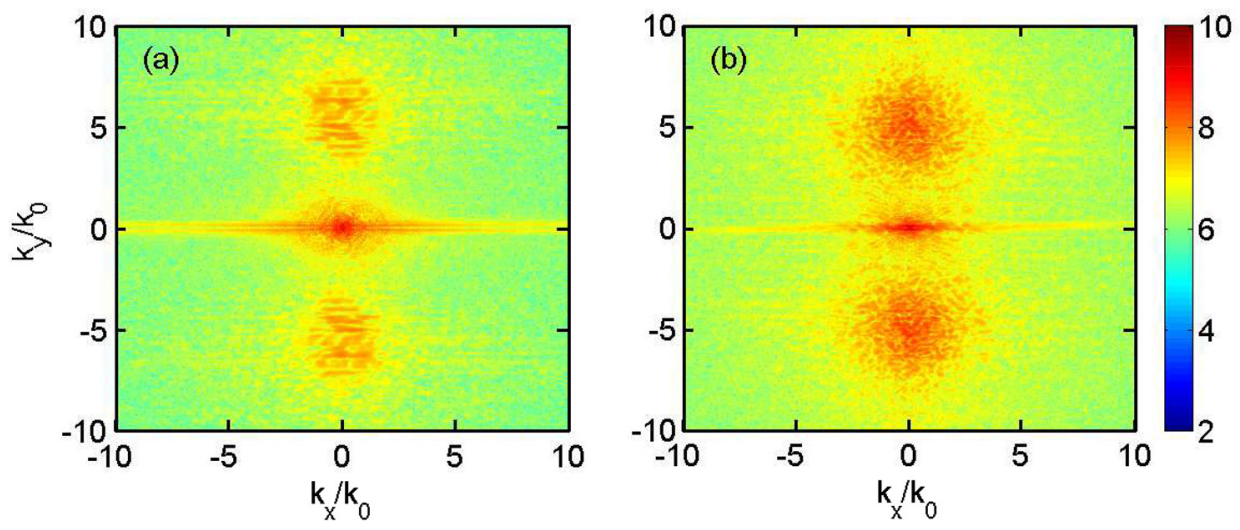

FIG. 3. Fast Fourier transform of $B_{z}$ at $\mathrm{t}=100 \mathrm{fs}$ (a) and $160 \mathrm{fs}(\mathrm{b})$, respectively. 
that electromagnetic oblique modes can be enhanced by collisional effects and FI is the dominant mode for a beam propagating in dense plasmas. Note that the peak around $\mathbf{k}=0$ is attributed to the fact that $\mathbf{k}$ becomes 0 as the laser pulse arrives at the critical point of the target.

For comparison, ultraintense laser interaction with a collisionless $\mathrm{Cu}$ target is also investigated, as shown in Fig. 4. It is seen that, large scale magnetic fields having distinct polarities are generated in the preplasma and around the solid target interface, which is similar to that in the collisional case. However, periodic magnetic field is not obvious in the collisionless plasma bulk, indicating that the plasma only experiences very weak instability growth, which is consistent with the results reported in Ref. 21 that collisionless FI is completely shut down for a RE beam with a large transverse temperature. The instability stabilized by the RE transverse energy spread because of beam transverse temperature has already been mentioned in many previous literatures. ${ }^{20,37,38}$ From the RE divergence, it can be estimated that the transverse temperature of REs is $480 \mathrm{keV}$ and a ratio of $T_{y} / T_{x}$ about 0.16 , where $T_{x}$ and $T_{y}$ are the RE temperature in the $x$ and $y$ directions, respectively, suggesting that transverse energy of the REs spreads remarkably during the RE propagating in the target. That is, the collision guarantees the occurrence of FI regardless of beam transverse temperature here. On the other hand, in the collisionless plasma, the FI would be suppressed significantly as the ratio of beam to plasma density gets very small. ${ }^{24}$ From the comparison, it is also predicted that the growth rate of the instability increases with the collision frequency in the case of RE beam and small ratio of beam to plasma. The REs propagate mainly in the forward direction in the collisionless case, as shown in Figs. 4(c) and 4(d), while they propagate forward with a parabolic profile in the collisional case (Figs. 1(c) and 1(d)), indicating that the REs should have smaller divergence in the former case compared to that of the latter. It is noted that the RE distribution is more diffuse than that in Fig. 1, which could be due to the absence of confinement of the FI induced magnetic fields that scatter the REs but can also confine the latter due to its very high intensity and wide spread.

Figure 5(a) shows the energy spectra of the electrons both for the collisional and collisionless $\mathrm{Cu}$ targets. It is shown that the spectra have similar profiles in these cases. The RE temperature is $3.03 \mathrm{MeV}$ for the collisional target and is identical to that of the collisionless case. It can be attributed to the fact that the REs here are mainly accelerated by the $\mathbf{J} \times \mathbf{B}$ heating, ${ }^{39}$ which can be clearly seen from the electron kinetic energy density distributions (Figs. 1 and 4), where the REs are separated by a distance of half of a wavelength, so the electron temperature is weakly affected by the collision frequency. The laser absorption efficiency for the collisionless case is nearly identical to that of the collisional case $(\sim 0.38)$. However, the number of the REs for the collisional case is a bit lower than that of the collisionless case due to the microscale magnetic fields arisen from the filamentation instability. Note that the RE temperature here is lower than that given by ponderomotive scaling ${ }^{40}$ $T_{h}=m_{e} c^{2}\left(\sqrt{1+a_{L}^{2}}-1\right)=4.14 \mathrm{MeV}$, which can be attributed to the fact that the electrons only interact with the laser pulse during a fraction of the laser cycle before being accelerated forward beyond the laser penetration region due to the relatively short preplasma scale-length employed in our simulation. $^{41}$

Angular distribution of REs $\left(E_{k} \geq 50 \mathrm{keV}\right)$ is shown in Fig. 5(b). The electron momentum angle here is determined by $\theta=\tan ^{-1}\left(p_{y} / p_{x}\right)$, where $p_{y}$ and $p_{x}$ are the electron momentum in the $y$ and $x$ directions, respectively. Since the relatively short length for target employed in the simulations,
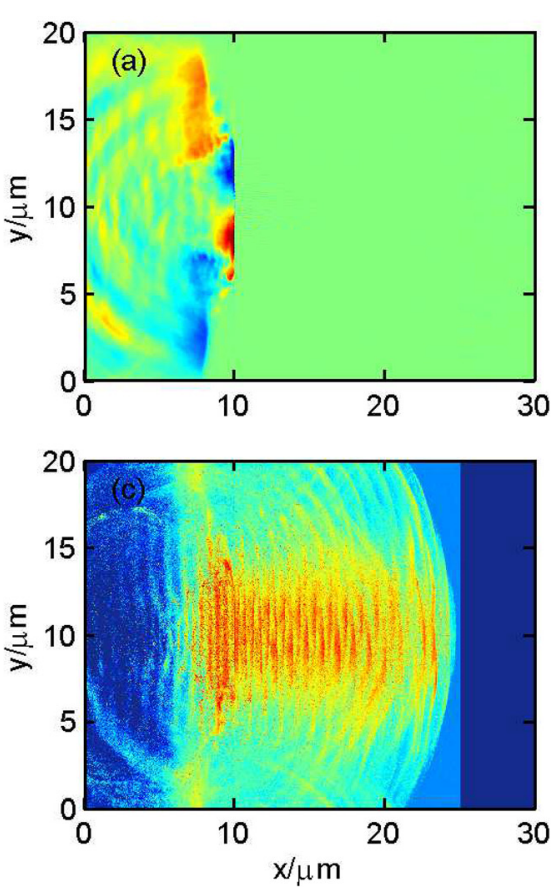
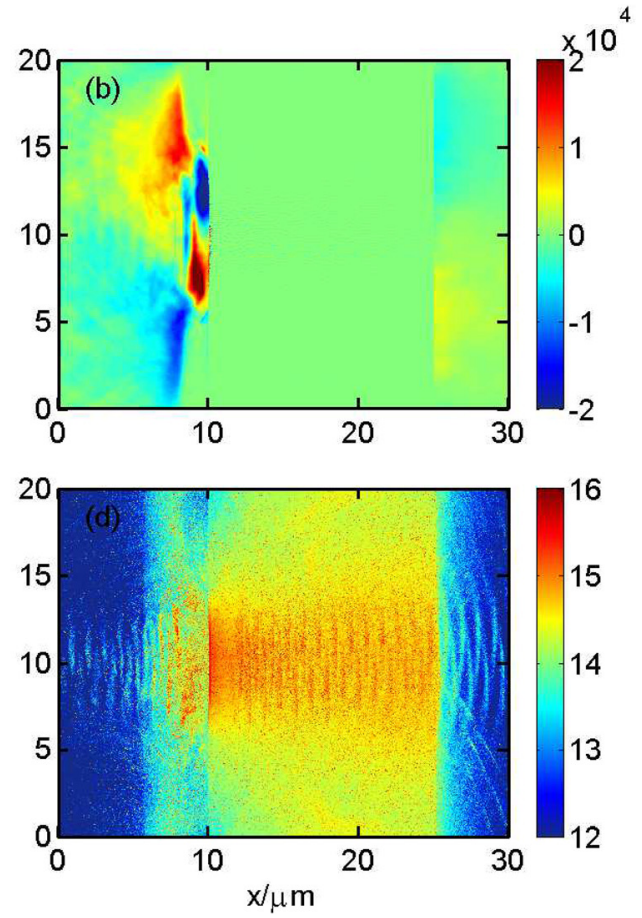

FIG. 4. The same as that in Fig. 1, but for a collisionless $\mathrm{Cu}$ target. 

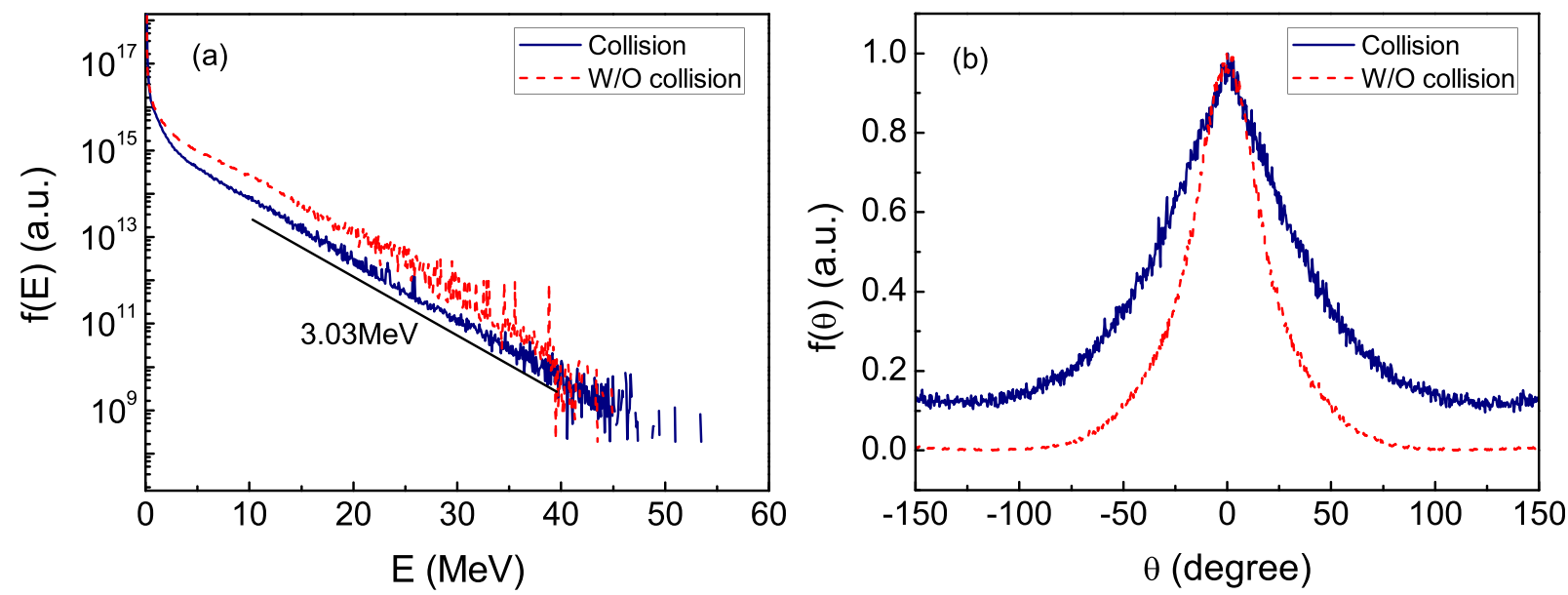

FIG. 5. Energy spectra of the electrons for collisional and collisionless Cu targets (a). Angular distribution of REs $\left(E_{k} \geq 50 \mathrm{keV}\right)$ for the collisional and collisionless $\mathrm{Cu}$ targets at $\mathrm{t}=100 \mathrm{fs}(\mathrm{b})$, respectively, in which the electrons are extracted from the region of $x=11-24 \mu \mathrm{m}$ and $y=4-16 \mu \mathrm{m}$ (the same as in Figs. 6(c) and 7(c)).

we mainly focus on the RE divergence at $t=100$ fs to avoid refluxing of REs from the rear side of the target. The electron angular distribution can be fitted by a Gaussian function

$$
f_{F}(\theta)=f_{0}+\frac{A}{\sigma \sqrt{\pi / 2}} e^{-2 \frac{\left(\theta-\theta_{c}\right)^{2}}{\sigma^{2}}},
$$

where $f_{0}$ and $A$ are constants, and $\theta_{c}$ and $\sigma$ are electron mean propagation angle and dispersion angle, which are related to the beam transverse velocity and the electron transverse temperature, respectively. It is shown that the full width at half maximum (FWHM) of the RE divergence reaches $66.9^{\circ}$ for the collisional case, which is significantly greater than that of the collisionless case $\left(43.4^{\circ}\right)$. This increase is attributed to the fact that the collisional FI induced magnetic field effectively scatters the REs. ${ }^{14}$ In the presence of such microscale magnetic field, the mean transverse velocity of the electrons is zero, but the mean square velocity is not, which determines the mean square angular of the electrons and leads to a large electron divergence. ${ }^{14}$

Figures 6(a) and 6(b) show the profile of $B_{z}$ along the $y$ direction at $x=11 \mu \mathrm{m}$ and the corresponding frequency spectrum $\left|B_{k}\right|$ of $B_{z}$ for the case with a laser intensity of $10^{19} \mathrm{~W} /$ $\mathrm{cm}^{2}$. It can be seen that the magnetic field here is relatively weaker with an amplitude less than $500 \mathrm{~T}$, and there is no obvious wave number peak in its frequency spectrum, meaning that the growth of filamentation instability is very tiny. It is due to the fact that lower RE current density is produced for the lower laser intensity compared to that of the high laser intensity. The laser absorption efficiency here is 0.48 , which is slightly higher than that of the aforementioned case. The dependence of RE divergence on laser intensity is presented in Fig. 6(c). Since the magnitude of the self-generated magnetic field increases with laser intensity due to higher RE current generation, it can scatter the REs more effectively. It is shown that the divergence increases with laser intensity, which is in good agreement with the experimental results. ${ }^{10}$ This is different from the results ${ }^{42}$ that higher laser intensity leads to smaller RE divergences, where only the divergence of much higher energy electrons is considered. It is also distinct from that in collisionless cases, in which the divergence of REs is weakly dependent on the laser intensity (not shown for brevity). The discrepancy indicates that the properties of the target should be considered comprehensively for investigating RE characteristics, especially for the solid target, in which the collision could have a significant influence on the RE divergence.

For comparison, the RE divergence for cases of ultraintense laser interaction with various materials is also studied,
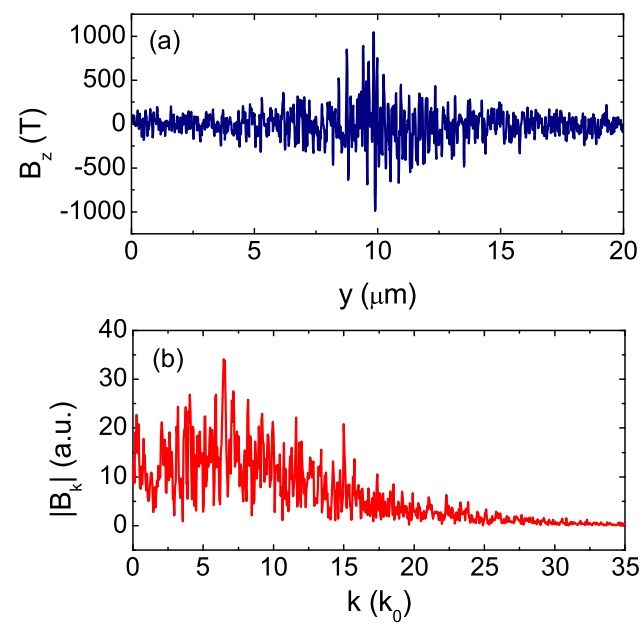

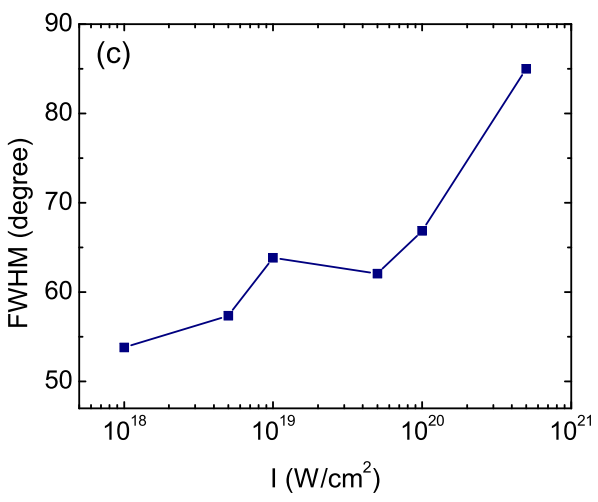

FIG. 6. The same as that in Fig. 2, but for a laser intensity of $10{ }^{19} \mathrm{~W} / \mathrm{cm}^{2}[(\mathrm{a})$ and (b)]. FWHM of the RE $\left(E_{k} \geq 50 \mathrm{keV}\right)$ angular distribution as a function of laser intensity for a collisional $\mathrm{Cu}$ target (c). 

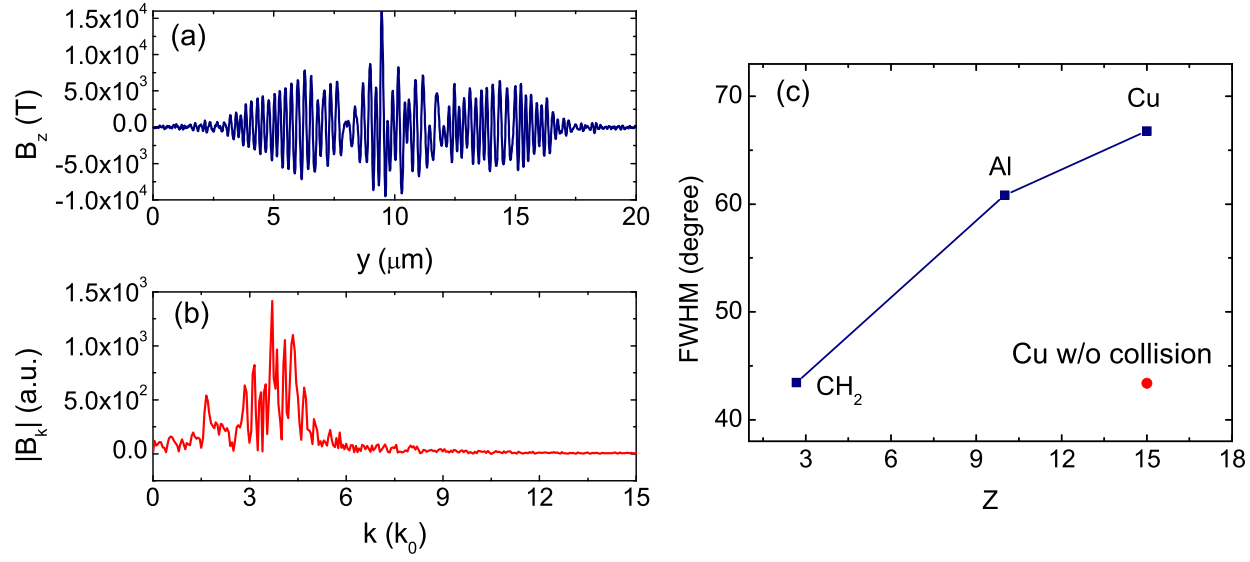

FIG. 7. The same as that in Fig. 2, but for a collisional $\mathrm{CH}_{2}$ target [(a) and (b)]. FWHM of the RE $\left(E_{k} \geq 50 \mathrm{keV}\right)$ angular distribution for the cases of collisional $\mathrm{Cu}, \mathrm{Al}$, and $\mathrm{CH}_{2}$ targets (c). The laser intensity is fixed at $10^{20} \mathrm{~W} / \mathrm{cm}^{2}$. as shown in Fig. 7. The charge state and maximum ion density for $\mathrm{CH}_{2}$ and $\mathrm{Al}$ targets are set to $2.67,10,45 n_{c}$, and $25 n_{c}$, respectively. In order to keep an identical target density that generates REs, similar preplasma profiles to that in the $\mathrm{Cu}$ target are used for both $\mathrm{CH}_{2}$ and $\mathrm{Al}$ targets. Note that the energy spectrum profiles of the electrons are very close to each other in these three cases (not shown for brevity), suggesting that the effect of the electron temperature differences for different targets can be neglected in this investigation. This is different with the results reported in Ref. 43 that the RE electron temperature is dependent on the target materials, in which a much lower laser intensity of $10^{16} \mathrm{~W} / \mathrm{cm}^{2}$ is employed, and the electrons are mainly accelerated by the resonance acceleration or vacuum heating (if the scale length of preplasma is less than the wavelength). ${ }^{42}$ In these regimes, the RE temperature is dependent on the scale length of the preplasma, which is different for different material targets because the plasma hydrodynamic expansion from lower- $Z$ targets is faster than that of high-Z targets. From Figs. 7(a) and 7(b), it is seen that microscale magnetic field similar to that in Fig. 2 is generated in the $\mathrm{CH}_{2}$ target, while the magnetic field is relatively weak $(5500 \mathrm{~T})$. The peak of the wave number is $\sim 3.7 k_{0}$, corresponding to a wave length of $5.5 \lambda_{p}$, slightly larger than that of the $\mathrm{Cu}$ target. The $\mathrm{RE}$ divergence for the $\mathrm{CH}_{2}$ target is only $43.4^{\circ}$ due to the relatively weak magnetic field in the target, which is equal to that in the collisionless $\mathrm{Cu}$ target. Figure 7(c) shows that the divergence increases rapidly with target charge state. It is because of that the collision frequency of electron-ion given by ${ }^{44} \nu_{e i}=\frac{4 \sqrt{2 \pi}}{3} \frac{n_{i} Z_{i}^{2} e^{4}}{m_{e}^{1 / 2} T_{e}^{3 / 2}} \ln \Lambda$ increases with plasma density and charge state, where $n_{i}$ and $Z_{i}$ are the ion density and charge state and $\ln \Lambda$ is the Coulomb logarithm. That is, the particles would experience higher collisions in high- $Z$ targets compared to that in low- $Z$ targets. Since the growth of FI in the case of extremely asymmetric counterstreaming is significantly suppressed only except that plasma collisions are considered, ${ }^{24}$ as observation in our simulations, stronger magnetic field generated and larger $\mathrm{RE}$ divergence induced in the $\mathrm{Cu}$ target compared to the $\mathrm{Al}\left(\right.$ or $\left.\mathrm{CH}_{2}\right)$ target.

\section{CONCLUSION}

In conclusion, angular distribution of laser-driven REs with laser intensity varying from $10^{18} \mathrm{~W} / \mathrm{cm}^{2}$ up to $5 \times 10^{20} \mathrm{~W} / \mathrm{cm}^{2}$ and target materials of $\mathrm{Cu}, \mathrm{Al}$, and $\mathrm{CH}_{2}$ are studied by collisional PIC simulations. Intense microscale magnetic field is generated by the FI during the REs propagating in collisional plasmas, and such instability is almost suppressed by the transverse temperature of REs (also due to the small ratio of beam to plasma density) in the collisionless target, leading to a great enhancement of the RE divergence in collisional plasmas compared to that of collisionless cases. The divergence of REs increases with laser intensity and target charge state due to the intense magnetic field generation for higher laser intensities and high- $Z$ targets. The results should be helpful for applications of laser-driven electron beams.

\section{ACKNOWLEDGMENTS}

This work was supported by the NNSFC (Nos. 11305264, 11275269, 11375265, and 91230205) and the Research Program of NUDT. The authors wish to acknowledge Professor M. Y. Yu (ZJU) and Dr. H. Schmitz (STFC RAL) for fruitful discussions.

${ }^{1}$ X. H. Yang, W. Yu, H. Xu, M. Y. Yu, Z. Y. Ge, B. B. Xu, H. B. Zhuo, Y. Y. Ma, F. Q. Shao, and M. Borghesi, Appl. Phys. Lett. 106, 224103 (2015).

${ }^{2}$ H. B. Zhuo, Z. L. Chen, Z. M. Sheng, M. Chen, T. Yabuuchi, M. Tampo, M. Y. Yu, X. H. Yang, C. T. Zhou, K. A. Tanaka, J. Zhang, and R. Kodama, Phys. Rev. Lett. 112, 215003 (2014).

${ }^{3}$ M. Tabak, J. Hammer, M. E. Glinsky, W. L. Kruer, S. C. Wilks, J. Woodworth, E. M. Campbell, M. D. Perry, and R. J. Mason, Phys. Plasmas 1, 1626 (1994).

${ }^{4}$ X. H. Yang, M. Borghesi, and A. P. L. Robinson, Phys. Plasmas 19, 062702 (2012).

${ }^{5}$ H. Shiraga, H. Nagatomo, W. Theobald, A. A. Solodov, and M. Tabak, Nucl. Fusion 54, 054005 (2014).

${ }^{6}$ A. P. L. Robinson, D. J. Strozzi, J. R. Davies, L. Gremillet, J. J. Honrubia, T. Johzaki, R. J. Kingham, M. Sherlock, and A. A. Solodov, Nucl. Fusion 54, 054003 (2014).

${ }^{7}$ A. Macchi, M. Borghesi, and M. Passoni, Rev. Mod. Phys. 85, 751 (2013).

${ }^{8}$ X. H. Yang, Y. Y. Ma, F. Q. Shao, H. Xu, M. Y. Yu, Y. Q. Gu, T. P. Yu, Y. Yin, C. L. Tian, and S. Kawata, Laser Part. Beams 28, 319 (2010).

${ }^{9}$ Z. Jin, Z. L. Chen, H. B. Zhuo, A. Kon, M. Nakatsutsumi, H. B. Wang, B. H. Zhang, Y. Q. Gu, Y. C. Wu, B. Zhu, L. Wang, M. Y. Yu, Z. M. Sheng, and R. Kodama, Phys. Rev. Lett. 107, 265003 (2011).

${ }^{10}$ J. S. Green, V. M. Ovchinnikov, R. G. Evans, K. U. Akli, H. Azechi, F. N. Beg, C. Bellei, R. R. Freeman, H. Habara, R. Heathcote, M. H. Key, J. A. King, K. L. Lancaster, N. C. Lopes, T. Ma, A. J. MacKinnon, K. Markey, A. McPhee, Z. Najmudin, P. Nilson, R. Onofrei, R. Stephens, K. Takeda, K. A. Tanaka, W. Theobald, T. Tanimoto, J. Waugh, L. Van Woerkom, N. C. Woolsey, M. Zepf, J. R. Davies, and P. A. Norreys, Phys. Rev. Lett. 100, 015003 (2008). 
${ }^{11}$ K. L. Lancaster, J. S. Green, D. S. Hey, K. U. Akli, J. R. Davies, R. J. Clarke, R. R. Freeman, H. Habara, M. H. Key, R. Kodama, K. Krushelnick, C. D. Murphy, M. Nakatsutsumi, P. Simpson, R. Stephens, C. Stoeckl, T. Yabuuchi, M. Zepf, and P. A. Norreys, Phys. Rev. Lett. 98, 125002 (2007).

${ }^{12}$ V. M. Ovchinnikov, D. W. Schumacher, M. McMahon, E. A. Chowdhury, C. D. Chen, A. Morace, and R. R. Freeman, Phys. Rev. Lett. 110, 065007 (2013).

${ }^{13}$ C. I. Moore, J. P. Knauer, and D. D. Meyerhofer, Phys. Rev. Lett. 74, 2439 (1995).

${ }^{14}$ J. C. Adam, A. Héron, and G. Laval, Phys. Rev. Lett. 97, 205006 (2006).

${ }^{15}$ A. Debayle, J. J. Honrubia, E. d'Humires, and V. T. Tikhonchuk, Phys. Rev. E 82, 036405 (2010).

${ }^{16}$ R. C. Davidson, D. A. Hammer, I. Haber, and C. E. Wagner, Phys. Fluids 15, 317 (1972).

${ }^{17}$ T. Y. B. Yang, J. Arons, and A. B. Langdon, Phys. Plasmas 1, 3059 (1994).

${ }^{18}$ T. Okada and K. Ogawa, Phys. Plasmas 14, 072702 (2007).

${ }^{19}$ F. Pérez, A. J. Kemp, L. Divol, C. D. Chen, and P. K. Patel, Phys. Rev. Lett. 111, 245001 (2013).

${ }^{20}$ K. Molvig, Phys. Rev. Lett. 35, 1504 (1975).

${ }^{21}$ A. Karmakar, N. Kumar, G. Shvets, O. Polomarov, and A. Pukhov, Phys. Rev. Lett. 101, 255001 (2008).

${ }^{22}$ L. Gremillet, G. Bonnaud, and F. Amiranoff, Phys. Plasmas 9, 941 (2002).

${ }^{23}$ C. A. Kapetanakos, Appl. Phys. Lett. 25, 484 (1974).

${ }^{24}$ B. Hao, Z. M. Sheng, C. Ren, and J. Zhang, Phys. Rev. E 79, 046409 (2009).

${ }^{25}$ L. Volpe, D. Batani, A. Morace, and J. J. Santos, Phys. Plasmas 20, 013104 (2013).

${ }^{26}$ X. H. Yang, H. B. Zhuo, Y. Y. Ma, H. Xu, T. P. Yu, D. B. Zou, Z. Y. Ge, B. B. Xu, Q. J. Zhu, F. Q. Shao, and M. Borghesi, Plasma Phys. Controlled Fusion 57, 025011 (2015).

${ }^{27}$ H. Schmitz, R. Lloyd, and R. G. Evans, Plasma Phys. Controlled Fusion 54, 085016 (2012).

${ }^{28}$ A. Héron and J. C. Adam, Phys. Plasmas 22, 072306 (2015).
${ }^{29}$ T. D. Arber, K. Bennett, C. S. Brady, A. Lawrence-Douglas, M. G. Ramsay, N. J. Sircombe, P. Gillies, R. G. Evans, H. Schmitz, A. R. Bell, and C. P. Ridgers, Plasma Phys. Controlled Fusion 57, 113001 (2015).

${ }^{30}$ Y. Sentoku and A. J. Kemp, J. Comput. Phys. 227, 6846 (2008).

${ }^{31}$ A. R. Bell, F. N. Beg, Z. Chang, A. E. Dangor, C. N. Danson, C. B. Edwards, A. P. Fews, M. H. R. Hutchinson, S. Luan, P. Lee, P. A Norreys, R. A. Smith, P. F. Taday, and F. Zhou, Phys. Rev. E 48, 2087 (1993).

${ }^{32} \mathrm{P}$. Gibbon, Short Pulse Laser Interaction with Matter: An Introduction (Imperial College Press, London, 2005).

${ }^{33}$ Y. Sentoku, K. Mima, S. Kojima, and H. Ruhl, Phys. Plasmas 7, 689 (2000).

${ }^{34}$ A. Bret, L. Gremillet, and M. Dieckmann, Phys. Plasmas 17, 120501 (2010).

${ }^{35}$ L. Gremillet, D. Bénisti, E. Lefebvre, and A. Bret, Phys. Plasmas 14, 040704 (2007).

${ }^{36}$ B. Hao, W. J. Ding, Z. M. Sheng, C. Ren, X. Kong, J. Mu, and J. Zhang, Phys. Plasmas 19, 072709 (2012).

${ }^{37}$ M. Fiore, F. iúza, M. Marti, R. A. Fonseca, and L. O. Silva, J. Plasma Phys. 76, 813 (2010).

${ }^{38}$ L. O. Silva, R. A. Fonseca, J. W. Tonge, W. B. Mori, and J. M. Dawson, Phys. Plasmas 9, 2458 (2002).

${ }^{39}$ W. L. Kruer and K. Estabrook, Phys. Fluids 28, 430 (1985).

${ }^{40}$ S. C. Wilks, W. L. Kruer, M. Tabak, and A. B. Langdon, Phys. Rev. Lett. 69, 1383 (1992).

${ }^{41}$ M. G. Haines, M. S. Wei, F. N. Beg, and R. B. Stephens, Phys. Rev. Lett. 102, 045008 (2009).

${ }^{42}$ Y. Q. Cui, W. M. Wang, Z. M. Sheng, Y. T. Li, and J. Zhang, Plasma Phys. Controlled Fusion 55, 085008 (2013).

${ }^{43}$ Z. L. Chen, J. Zhang, T. J. Liang, H. Teng, Q. L. Dong, Y. T. Li, J. Zhang, Z. M. Sheng, L. Z. Zhao, and X. W. Tang, J. Phys. B: At. Mol. Opt. Phys. 37, 539 (2004).

${ }^{44}$ T. C. Ma, X. W. Hu, and Y. H. Chen, Principle of Plasma Physics (University of Science and Technology of China Press, Hefei, China, 2012). 